\title{
Simulação em manequins como estratégia de ensino-aprendizagem para avaliação de ferida: relato de experiência
}

\author{
Simulation with mannequins as a learning-teaching strategy for wound care \\ assessment: an experience report
}

\section{Maniquíes de simulación como estrategia de enseñanza-aprendizaje para la evaluación de la herida: un relato de experiencia}

\author{
Belayrla Cerqueira de Jesus ${ }^{1}$, Gislaine Felix Ramos ${ }^{1}$, Cristiane Costa Reis da Silva², \\ Virginia Crispina Oliveira Gomes ${ }^{3}$, Gilberto Tadeu Reis da Silva ${ }^{3}$
}

\begin{abstract}
RESUMO
A simulação realística é um instrumento de ensino e avaliação e oferece diversas vantagens para o aprendizado de práticas básicas e complexas. A simulação com o uso de manequins permite que estudantes aprimorem suas habilidades clínicas sem expor o paciente a erros, o que traz mais segurança aos discentes e usuários. Na enfermagem, entre os diversos procedimentos que requerem o treinamento de graduandos encontra-se a técnica de curativo no tratamento de feridas, ainda difícil de ser realizada de maneira eficaz, confortável ao paciente e esteticamente aceitável, tornando assim fundamental o ensino dos estudantes. O objetivo do presente estudo foi descrever a utilização de uma estratégia de ensino focada na simulação realística aplicada para avaliação de feridas em uma instituição de ensino superior no estado da Bahia. Relato de experiência que descreve aspectos vivenciados pelos discentes nas situações simuladas. Conclui-se que a simulação como estratégia de ensino-aprendizagem para avaliação de feridas é considerada um apoio didático, que possibilita praticar procedimentos clínicos em um espaço seguro e muito próximo do real, sem risco de exposição do paciente.
\end{abstract}

DESCRITORES: Estomaterapia. Simulação. Ensino. Enfermagem.

\begin{abstract}
Realistic simulation is a teaching and evaluation tool that offers several advantages for learning basic and complex practices. The simulation with the use of dummies allows students to improve their clinical skills without exposing the patient to errors, which brings more security to students and users. In nursing, among the various procedures that require graduate training there is the dressing technique in the treatment of wounds. This is yet difficult to achieve in an effective, comfortable and aesthetically acceptable way to the patient, thereby becoming fundamental to teach students. The objective of this study was to describe the students' experience in the use of teaching strategies focused on realistic simulation applied to evaluate wounds in a higher education institution in the state of Bahia, Brazil. It is an experience report describing aspects experienced by students in simulated situations. We conclude that simulation, as a teaching and learning strategy for evaluating wounds, is considered a didactic support, which enables practicing clinical procedures in a safe way very close to the real situation, without the risk of patient exposure.
\end{abstract}

KEYWORDS: Stomatherapy. Simulation. Teaching. Nursing.

\footnotetext{
${ }^{1}$ Faculdade Ruy Barbosa DeVry - Salvador (BA), Brasil.

¿Universidade Salvador (UNIFACS); Centro Universitário Jorge Amado (UNIJORGE) - Salvador (BA), Brasil.

${ }^{3}$ Universidade Federal da Bahia (UFBA) - Salvador (BA), Brasil.

Endereço para correspondência: Cristiane Costa Reis da Silva - Rua Joaquim Calado, 120 - Federação - CEP: 4023-0093 - Salvador (BA), Brasil -

E-mail:cristianereisfb@gmail.com

Artigo recebido em: 25/09/2014 - Aceito para publicação em: 27/05/2016
} 


\section{RESUMEN}

Simulación realística es un instrumento de enseñanza y evaluación y ofrece diversas ventajas para el aprendizaje de prácticas básicas y complejas. La simulación con el uso de maniquíes permite que los estudiantes mejoren sus habilidades clínicas sin exponer al paciente a errores, lo que da más seguridad a los estudiantes y usuarios. En la enfermería, entre los diversos procedimientos que requieren el entrenamiento de graduandos se encuentra la técnica de curativo en el tratamiento de heridas, aun difícil de ser realizada de manera eficaz, confortable para el paciente y estéticamente aceptable para el paciente, volviendo así fundamental la enseñanza a los estudiantes. El objetivo del presente estudio fue describir la utilización de una estrategia de enseñanza enfocada en la simulación realística aplicada a la evaluación de heridas en una institución de educación superior en el estado de Bahía. Relato de experiencia que describe aspectos vivenciados por los estudiantes en las situaciones simuladas. Se concluye que la simulación como estrategia de enseñanza y aprendizaje para evaluación de heridas es considerada un apoyo didáctico, que permite la práctica de procedimientos clínicos en un espacio seguro y muy cerca de la realidad, sin riesgo de exposición del paciente.

DESCRIPTORES: Estomaterapia. Simulación. Enseñanza. Enfermería.

\section{INTRODUÇÃO}

O centro de simulação realística foi inaugurado em 2013, em uma Instituição de Ensino Superior (IES) privada, localizada no município de Salvador, estado da Bahia, com base no projeto Turbo Saúde, criado em 2012 em outra IES do mesmo grupo. Teve como objetivo melhorar o aprendizado dos alunos, utilizando laboratórios equipados para simulação. Considerando que os estudantes da área de saúde necessitam de treinamento de habilidades, simples ou complexas, torna-se vantajoso inserir atividades de ensino por meio da simulação em bonecos, a fim de proporcionar a reprodução dos procedimentos com uma formação de alta qualidade ${ }^{1}$.

Nos últimos anos, grandes investimentos têm sido feitos no cenário educacional brasileiro. A busca por metodologias inovadoras e estratégias de ensino que promovam aos estudantes e usuários do setor da saúde maior segurança tem sido objeto de vários estudos. Esse movimento e tendência das práticas pedagógicas sinalizam uma mudança no ensino superior em saúde e, por conseguinte, no de enfermagem. O uso da simulação realística durante o processo de formação dos profissionais dessa área tem permitido que desenvolvam habilidades necessárias ao seu crescimento profissional, com a vantagem, nesse momento, de poderem errar. As mudanças na prática clínica, como a redução do período de internação, tornaram menos viável a utilização de pessoas doentes como recurso pedagógico e levaram à necessidade de novos métodos de instrução, aquisição de conhecimentos e avaliação. A simulação, como instrumento de ensino e avaliação, oferece diversas vantagens para o aprendizado de práticas básicas, como a realização de curativos e suas técnicas ${ }^{2}$.

Quando se almeja o ensino de habilidades simples, simuladores de baixa tecnologia podem ser utilizados, por exemplo, como modelos anatômicos que permitam a exploração e o aprendizado de procedimentos pertinentes à enfermagem. O uso de manequins de procedimentos no processo de simulação propicia o desenvolvimento de habilidades psicomotoras, $\mathrm{o}$ que torna os estudantes mais confiantes e seguros no momento de prestar assistência ao paciente ${ }^{3}$.

A simulação com o uso de manequins é vista como importante estratégia para evitar erros e a exposição desnecessária dos pacientes, porém, o aprendizado de habilidades inviáveis de serem trabalhadas com o manequim, como reações fisiológicas, capacidade de comunicação e tratamento humanizado, ocorrerá na prática clínica ${ }^{4}$.

Durante o processo ensino-aprendizagem, em que o conhecimento é construído por meio de situações programadas, representativas da realidade prática profissional, simuladas por protótipos, os estudantes podem aprimorar suas habilidades clínicas sem arriscar a segurança dos usuários.

Diante do exposto, observando-se a importância que a simulação vem adquirindo no meio acadêmico,e com foco na técnica do curativo no tratamento de feridas, ainda difícil de ser realizada de maneira eficaz, confortável ao usuário e esteticamente aceitável, o presente estudo tem como objetivo descrever a utilização de uma estratégia de ensino focada na simulação realística aplicada para avaliação de feridas em uma IES no estado da Bahia.

\section{MÉTODOS}

Relato de experiência que descreve aspectos vivenciados pelos discentes nas situações simuladas. $O$ relato de experiência é uma ferramenta da pesquisa descritiva que permite reflexão sobre uma ação ou um conjunto de ações que tratam de uma situação vivenciada no âmbito profissional, de comum interesse da comunidade científica. 
A atividade avaliada neste estudo foi desenvolvida durante o ensino teórico-prático no componente curricular da disciplina de Procedimentos Básicos de Enfermagem, na aula Assistência de Enfermagem nas Lesões de Pele, ministrada em uma IES do município de Salvador, estado da Bahia, durante o período de seis meses.

O cenário representou um procedimento de avaliação de feridas, com duração média de 15 minutos, para análise da capacidade de desempenho do aluno. A estratégia foi realizada com 20 alunos do quarto semestre, no Laboratório de Habilidades. A turma foi dividida em cinco grupos, contendo quatro alunos cada. Enquanto dois alunos atuaram na simulação, os demais assistiram, anotando as observações; posteriormente foi feita a troca de papéis.

\section{Dinâmica da simulação}

A simulação realizada no Laboratório de Habilidades foi precedida de aula teórica, na qual foram abordadas: anatomia da pele, fisiologia e avaliação da ferida, tipos de feridas infectadas, assim como as principais técnicas de curativo, visando uma maior associação entre a prática e a teoria e desenvolver conhecimento técnico-científico.

No início da simulação foi apresentado um caso clínico, sendo descrita a situação hipotética de um paciente. O material disponibilizado pelo laboratório da instituição para essa atividade foi um modelo de glúteo (WoundcaremodelLaerdal) apresentando úlceras e incisão cirúrgica com deiscência, utilizado para avaliação da ferida e realização do curativo (Figuras 1 e 2). Em seguida, após avaliação da ferida, os materiais foram preparados para realização da técnica e os alunos orientados, pelos docentes presentes, de maneira clara e explicativa, sobre o procedimento que seria realizado, o qual se iniciou após higienização das mãos,

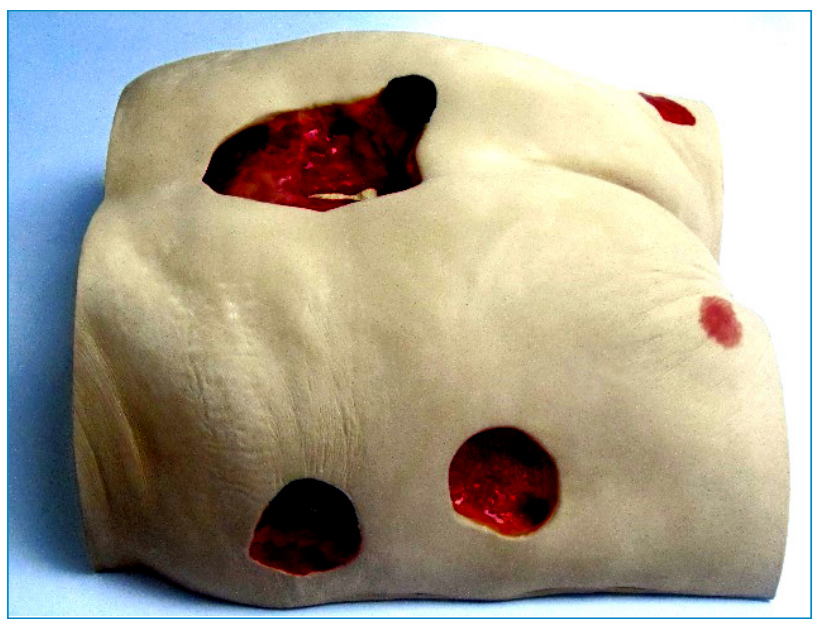

Figura 1. Modelo anatômico de feridas. imprescindível para manuseio e contato com o paciente. Procedeu-se então à irrigação da área lesada com solução fisiológica (SF) 0,9\% e à limpeza da ferida seguindo técnica asséptica.Posteriormente, a ferida foi avaliada no modelo anatômico (Figuras 3 e 4) quanto a extensão, bordas, localização,

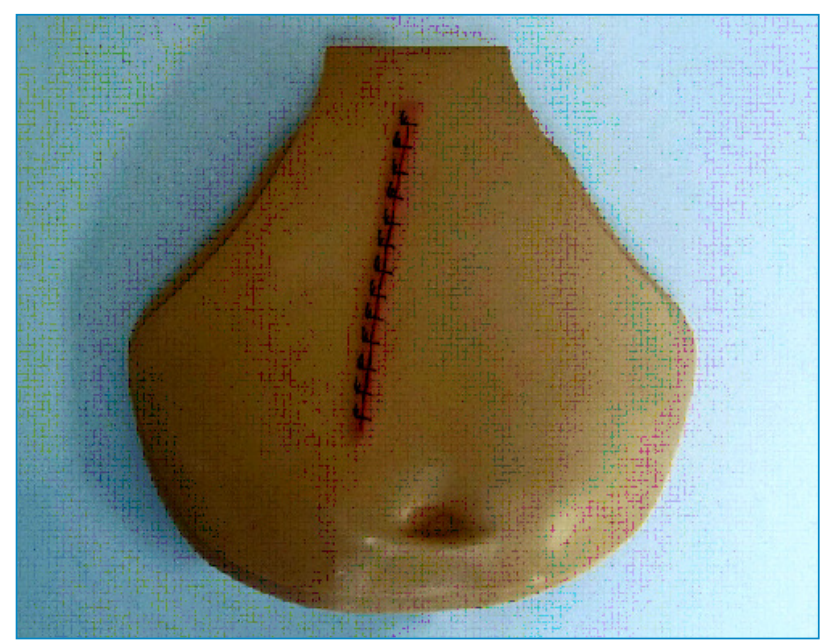

Figura 2. Incisão cirúrgica.

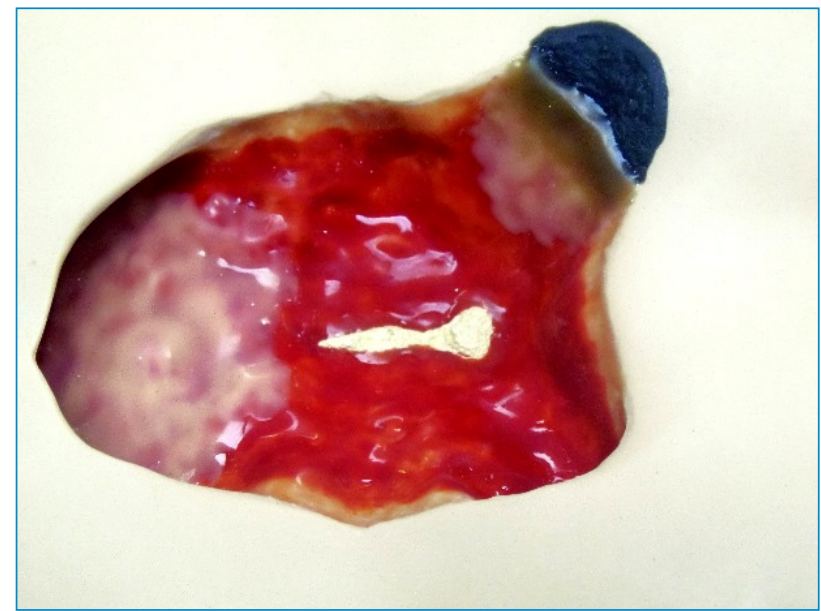

Figura 3. Modelo anatômico de lesão por pressão em região sacra.

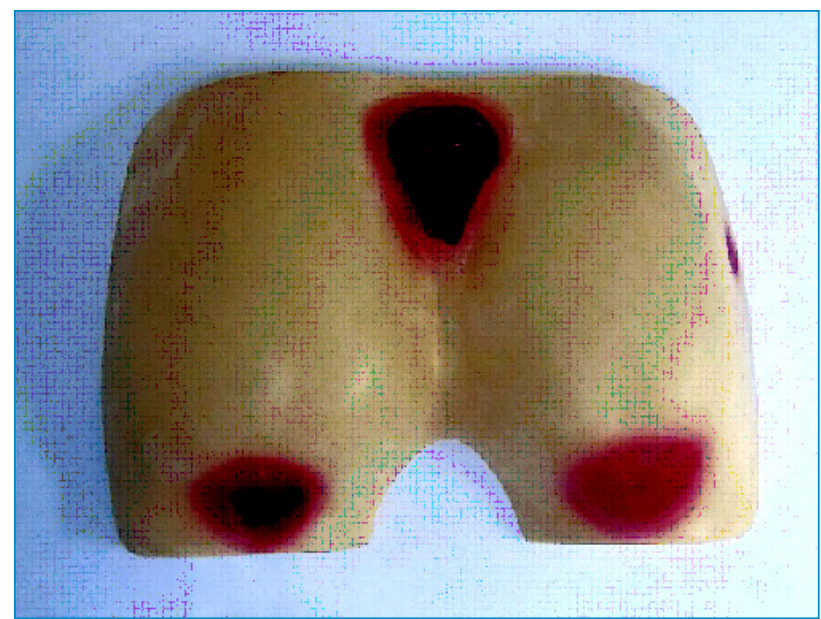

Figura 4. Modelo anatômico de lesão por pressão. 
presença de exsudato e tecido necrosado. Observou-se também o tipo de cicatrização.

A simulação teve duração de 15 minutos. Além da avaliação, a utilização do manequim de sutura oportunizou o treinamento da técnica em uma incisão cirúrgica.

Durante a simulação, os docentes utilizaram um instrumento do tipo checklist visando avaliar a técnica realizada, bem como identificar aspectos detectados nessa avaliação. Essa ferramenta possibilitou verificar o conhecimento do aluno desde a etapa de higienização das mãos, abordagem ao paciente, explicação sobre o procedimento a ser realizado, manipulação do material esterilizado, remoção do curativo, técnica, aspectos da lesão a serem observados, cobertura utilizada, descarte do material e registro no prontuário. Para avaliação da ferida, utilizou-se a mnemônica proposta por Keast et al. (apud Dealey ${ }^{5}$ ) (MENSURE), a qual inclui medida, exsudato, aparência, dor, descolamento, reavaliação e borda.

O desenvolvimento do estudo obedeceu aos preceitos éticos estipulados pela Resolução no 466/2012 do Conselho Nacional de Saúde, após aprovação do projeto pelo Comitê de Ética em Pesquisa através da Plataforma Brasil sob o registro no 1.139 .813 .

\section{RESULTADOS}

Os alunos que estavam assistindo à simulação participaram ativamente, expuseram os problemas encontrados e mencionaram falhas e sugestões para melhorar o atendimento. $\mathrm{O}$ docente, por sua vez, suscitou a discussão, analisou e refletiu com o grupo os objetivos das ações, benefícios, comportamentos dos participantes, entre outros. As aulas práticas em laboratório de simulação podem ser destacadas como importante fator de associação teórico-prática, visto que favorecem o aprendizado. Deve-se ressaltar também que, além da realização da técnica, foi possível reforçar condutas necessárias durante o preparo do material, tais como observação da data de validade das substâncias utilizadas para curativo da ferida, lacre, validade da esterilização dos materiais a serem utilizados, procedimento a ser realizado e a postura profissional. A importância de um registro adequado também foi pontuada como forma essencial de acompanhar a evolução do tratamento. Os registros de cuidados e ações de enfermagem devem ser realizados de forma sistemática, tendo como base instrumentos que facilitem as anotações das características das feridas, bem como dos fatores que podem adiar o processo de cicatrização ${ }^{6}$.

\section{DISCUSSÃO}

A técnica realizada em laboratório por meio da simulação foi considerada pelos alunos como algo mais realista, capaz de torná-los mais confiantes, seguros e habilidosos durante a realização do procedimento, o que também minimiza o receio de fazê-lo diretamente nos pacientes. Tem sido, portanto, apontada como estratégia facilitadora do aprendizado ${ }^{7,8}$.

Embora a simulação tenha sido precedida de aula sobre o assunto, observamos que o paciente não foi orientado quanto ao procedimento.

Outro fator relevante observado e que não foi mencionado pelos alunos foi a avaliação da dor, cujo aumento pode ser indicativo de infecção, o que torna fundamental que o profissional atente para esse aspecto durante sua abordagem. A medida da área lesada foi realizada em seu maior comprimento e largura, conforme preconizado na literatura ${ }^{9,10}$.

Ressalta-se que o monitoramento da ferida é importante para auxiliar no tipo de cobertura a ser utilizada, todavia, apesar de esse ponto ter sido citado pelos discentes na avaliação, o simulador utilizado não permite medir a quantidade de exsudato e o odor, porém, consideramos benéfica a pontuação desses itens. Acresce-se que no leito da ferida foi identificada necrose e, nesse caso, podemos considerar o modelo vantajoso, visto que foi útil para percebermos o descolamento do tecido necrótico.

De maneira geral, foi possível perceber que a estratégia de ensino por meio da simulação possibilitou e favoreceu a aprendizagem específica de tratamento de feridas.

A formação dos profissionais de saúde é um fator intimamente ligado à qualidade da assistência prestada e à segurança do paciente ${ }^{11}$. $\mathrm{O}$ treino de habilidades específicas com o uso da experiência clínica simulada representa um aspecto essencial para que essa situação possa ser fácil e integralmente entendida pelo estudante, permitindo uma resposta adequada quando algo semelhante acontecer em um contexto real ${ }^{12,13}$.

Segundo Martins ${ }^{14}$, o ambiente de simulação com cenários completos e complexos permite que os estudantes consolidem seus saberes e desenvolvam as capacidades de 
raciocínio crítico e tomada de decisão, além das competências técnicas, relacionais e éticas ${ }^{14}$.

Vários estudos apontam que o uso da simulação realística com vivências e experiências clínicas simuladas se tornou uma importante estratégia no ensino de enfermagem que pode ser utilizada tanto na formação de graduandos como de pós-graduandos na área, gerando amplos conhecimentos para os formandos, sobretudo, no que se refere ao desenvolvimento de competências para o raciocínio crítico e o estabelecimento de prioridades, a tomada de decisão, a realização de ações corretas, o trabalho em equipe e a correção de erros sem os efeitos desses nos pacientes.

\section{CONCLUSÃO}

Neste estudo foi possível observar que a simulação realística constitui método didático efetivo e inovador, auxiliando no processo ensino-aprendizagem por possibilitar ao aluno praticar procedimentos clínicos em um espaço pedagógico seguro, controlado e muito próximo do real, sem o risco da exposição do paciente.

A simulação realística do procedimento de curativo demonstrou-se vantajosa para o processo de ensino-aprendizagem, um método capaz de treinar os estudantes para a prática profissional, ressaltando que os discentes, após sua formação, se encontrarão preparados para atuar e prestar uma assistência de qualidade.

\section{REFERÊNCIAS}

1. Martins JCA, Mazzo A, Baptista RCN, Coutinho VRD, Godoy S, Mendes IAC, et al. A experiência clínica simulada no ensino de enfermagem: retrospectiva histórica. Acta Paul Enferm. 2012;25(4):619-25.

2. Amaral JMV. Simulação e ensino-apredizagem em pediatria. Ia Parte: tópicos essenciais. Acta Ped Port. 2010;41(1):44-50.

3. Gomes CO, Germano RM. Processo ensino/aprendizagem no laboratório de enfermagem: visão de estudantes. Rev Gaúcha Enferm. 2007;28(3):401-8.

4. Kruse MHL. É possível pensar de outro modo a educação em Enfermagem? Esc Anna Nery Rev Enferm. 2008;12(2):348-52.

5. Dealey C. Cuidando de feridas: um guia para as enfermeiras. 3. ed. São Paulo: Atheneu; 2008. p. 56-82.

6. Bajay HM, Pedrosa MMO, Lourenço MTN, Cortez SL, Paula MAB. Registro de avaliação e evolução de feridas: subsídios para reflexão e mudanças. Rev Estima. 2003;1(2):20-9.

7. Reilly A, Spratt C. The perceptions of undergraduate student nurses of high-fidelity simulation-based learning: a case report from the University of Tasmania. Nurse Educ Today. 2007;27(6):542-50.

8. BarsukJH, McGaghie WC, Cohen ER, O'Leary KJ, Wayne DB. Simulation-based mastery learning reduces complications during central venous catheter insertion in a medical intensive care unit. Crit Care Med. 2009;37(10):2697-701.

9. Nascimento AR, Namba M. Aspecto da ferida: avaliação de enfermagem. Rev Enferm UNISA. 2009;10(2):118-23.

10. Florianópolis. Prefeitura Municipal. Protocolo de Feridas da Prefeitura Municipal de Florianópolis. Florianópolis: Secretaria Municipal de Saúde; 2008.

11. Trevizan MA, Mendes IAC, Mazzo A, Ventura CAA. Investment in nursing human assets: education and minds of the future. Rev Latino-Am Enfermagem. 2010;18(3):467-71.

12. Nehering $W$. History of simulation in nursing. In: Nehring W, Lashley F, organizers. High-fidelity patient simulation in nursing education. Quebec: Jones and Bartlett Publishers; 2010.

13. Jeffries $P$, organizer. Simulation in nursing education: from conceptualization to evaluation. New York: National League for Nursing; 2007.

14. Martins J. Atuação do enfermeiro no setor de urgências: gestão para o desenvolvimento de competências. In: Malagutti W, Caetano C, organizadores. Gestão do serviço de enfermagem no mundo globalizado. Rio de Janeiro: Rubio; 2009. Capítulo 14. 